pyrexia, and Dr. FitzGibbon was invited to see the case, He expressed a definite opinion that the operation could be performed without risk, and further stated that he thought both the mental and physical phenomena were the result of septic absorption from the uterus.

\section{The Operation}

On October 7 th, 1931, a complete hysterectomy was performed, both ovaries being removed at the same time. The excised uterus was found to be in a much more septic condition than had been imagined. Dr. FitzGibbon reported that at operation there were no peritoneal adhesions, the uterus was retroverted, and the parametrium on the right side of the cervix was sclerotic and contracted. The whole uterus and cervix from the vaginal fornices with both tubes and ovaries were removed: tubes normal, atrophied ; ovaries small, atrophied, contained some chronic simple follicular cysts ; uterus $4 \frac{1}{2}$ inches long, walls of body 1 to $1 \frac{1}{4}$ inches thick; cervix was $1 \frac{3}{4}$ inches long-about $3 / 4$ inch vaginal portion, 1 inch supravaginal. Dr. Rowlette, who had an opportunity of seeing the specimen immediately after removal, reported as follows :

The uterus was much larger than normal, the enlargement being symmetrical and due to a general swelling rather than to the presence of a tumour. There was no fibroid or other tumour. The organ was soft and spongy, and did not present the appearance of a uterus the subject of fibrosis uteri, or show any obvious increase of fibrous tissue. The endometrium was inflamed-a dirty greyish green in colour, looking very unhealthy. In my opinion the organ was the subject of general metritis, affecting both the muscle and the endometrium, and subacute in course. To appreciate the degree of hyperplasia, the uterus should be compared with a normal organ after the menopause atrophy, which would be about $2 \frac{1}{2}$ inches long with no vaginal cervix, walls about $1 / 2$ inch thick, and all soft and flabby, with small blood vessels.

\section{Result of Operation}

From the physical point of view the patient stood the operation well, and, apart from a mild heart attack three weeks later, probably caused by small emboli reaching the lungs, she made an uninterrupted recovery. The most striking result of the operation, however, and that which leads to the publication of this note, is the fact that almost immediately after the operation her mental condition showed a most remarkable improvement. A week before the operation an experienced alienist regarded her condition as one of hopeless pre-senile dementia. Three weeks after the operation she was able to reply sensibly to simple questions. After a week she began to notice her surroundings and to recognize her friends and relatives. In a fortnight there was evidence of returning memory. From that day there has been progressive improvement physically and mentally. At the time of writing this note, five months after the operation, the patient is able to go out for daily walks and drives; she eats well, sleeps well, and enjoys the visits and conversation of her friends. She writes short letters, can read the newspaper for a short period, and her face has recovered its old expression of intelligence. She still tires easily, but continues to improve in every respect from week to week.

\section{Discussion}

It was unfortunate that in this case no bacteriological examination of the specimen was made, but the report of Dr. Rowlette, formerly pathologist to the Rotunda Hospital, leaves no doubt that the condition was one of chronic bacterial infection. Such infection would explain the pyrexial attacks from which the patient suffered and also the venous thrombosis. Further, it seems almost certain that the septic condition of the uterus was the cause of the mental symptoms. The actual result of the operation, from a therapeutic point of view, was more dramatic than it is possible to convey in words. Before the operation the patient was apparently hopelessly insane, and was rapidly passing into a condition of complete dementia. Immediately after the operation she began to improve, and the improvement, as above stated, has been progressive.

Dr. FitzGibbon, to whom the credit of effecting this cure is due, holds the opinion that many of the customary phenomena of the menopause can be averted or cured by removal of the uterus, even when no infection is present. $\mathrm{He}$ believes that in the process of involution of the uterus toxic products are formed in that organ which are absorbed into the blood and give rise to flushings and other symptoms. He further holds that if the uterus is enlarged or fibrotic, toxins will be formed in greater quantity than in normal individuals, and that more serious menopause symptoms are therefore likely to arise in these cases. $\mathrm{He}$ is a strong advocate of performing hysterectomy in all menopause cases when the uterus is enlarged, and in all cases when menopausal symptoms are severe.

To me Dr. FitzGibbon's view is entirely novel, and my own belief is that the cure brought about in the present case was due to the removal of a definitely septic focus ; but the result was so remarkable, and the operation so easily borne, that I feel it would be worth while giving a thorough trial to Dr. FitzGibbon's views in all cases of menopausal insanity, provided the services of an expert gynaecological surgeon can be obtained. The case at any rate suggests the advisability of dealing radically with all foci of septic infection in cases of menopausal or other insanity.

\section{THE MENOPAUSE AND ITS COMPLICATIONS}

GIBBON FITzGIBBON, M.D., F.R.C.P.I., F.C.O.G. GYNAECOLOGIST, MERCER'S HOSPITAL, DUBLIN

The case of menopausal disturbance cured by hysterectomy recorded by Dr. Moorhead (p. 923), in which the condition of the patient was compatible with a diagnosis of senile dementia, should suggest that the generally accepted ideas on the menopause are not quite correct. Dr. Moorhead indicates that I place a different interpretation on the clinical symptoms generally supposed to be due to the menopause from that usually accepted. It was owing to these views that I expressed the opinion $I$ did in Dr. Moorhead's case, and in this paper I wish to propound, and as far as possible explain, my own. For that purpose I must give my summary of the case, which was an extreme one of its type ; but my beliefs are not in any way based on it, although it adds considerable support to them.

\section{Dr. Moorhead's CASE}

I have been personally acquainted for many years with the patient. She was married when 22 years of age, and had one child soon after marriage. She acquired a retroversion of the uterus after her confinement and had two enrly abortions and a second child seven years after marriage. The uterus then remained in retroversion. There was evidence of septic infection at her confinement, but this cleared up, and the patient subsequently led a normal, active, healthy life. The monthly periods were always of the heavy type, and became more so from the age of 45 ; this, as usual, was considered to be associated with the approach of the menopause.

When 49 years of age curettage was performed on account of the menstrual loss, but without benefit; the uterus was found to be retroverted and enlarged. The possibility of a fibroid was considered, as well as hysterectomy; this was not done, as the existence of a fibroid was very doubtful, and the monthly periods continued to occur regularly. At 
this time the patient had considerable domestic responsibilities, which she willingly assumed, and always appeared indefatigable.

In July, 1930, at the age of 51 , she received a shock, and collapsed into a condition suggestive of a brain lesion. This was the beginning of her illness as described by Dr. Moorhead. The monthly periods continued regular, but heavy, until January, 1931, when a period of amenorrhoea set in and continued for six months. Then vaginal discharge appeared and the uterus was douched, a moderate degree of haematometra being discovered and removed. The next monthly period occurred after twenty-eight days; it was profuse, but not prolonged. A few weeks after this I first saw the patient. I found the uterus in retroversion; it was enlarged to three or four times the size of a normal non-pregnant uterus; the vaginal portion of the cervix projected about three-quarters of an inch into the vagina; and the supravaginal portion was very bulky, giving an indurated, nonelastic resistance. On the right side the parametrium was hard and indurated, making palpation through it difficult. There was a fairly profuse viscid leucorrhoeal discharge, with a very slight, if any, purulent element. In my opinion there was sufficient evidence of chronic induration of the supravaginal cervix and surrounding parametrium for these parts to be the centre of a chronic toxic absorption, capable of producing a very considerable degree, if not the whole, of the patient's general and mental debility. This opinion was based on my observations of the enormous improvement in the physical and mental state of several patients upon whom I had performed hysterectomy for very similar uterine states causing disturbance at or after the time of the menopause, and particularly of the two cases to be described.

\section{Two Other CAses}

1. A married woman, aged 66 years, last confinement at age of 26, was referred to me on account of slight but persistent haemorrhagic discharge from the vagina. Menstrual bleeding had ceased at the age of $\mathbf{5 2}$. Shortly after this she had lost a son during the great war, and since then had lost interest in everything, had developed a mild melancholia, complained of very little, objected to interference, and seemed merely to wish to be left alone. The uterus was removed by vaginal hysterectomy. Six months later I learned that my patient had resumed all her former social activities, and enjoyed seeing visitors, paying visits, going to entertainments, and even going for trips from home.

2. A widow, aged 48 years, with one child, 16 years old, had for the past two or three years suffered from debility, supposed to be due to menopausal bleeding. She was described by her relations as doing nothing but mope after her son. Five months after a hysterectomy had been performed she had assumed a cheerful mental aspect quite different from her former state, taking a keen interest in life generally, and enjoying outdoor sports.

I was much impressed by the physical and mental change in these two patients. Both had small fibroids of the uterus which ultimately indicated treatment by hysterectomy, but in neither was the uterine bleeding sufficient to produce the degree of physical and mental depression from which they suffered. In the former the menopause was delayed, but no bleeding had occurred for ten years, and then it was only a stained discharge. In the latter the menstrual loss was heavy; there was some anaemia, but not sufficient to make the patient seek advice. The removal of the whole uterus was followed in a short time by an unexpected and remarkable improvement in the physical and mental well-being of the patients.

On following up other patients treated by total hysterectomy during or after the menopause $I$ found similar marked improvement in the general and psychological conditions. I have subsequently performed hysterectomies on a number of women suffering from persistent ill-health of the varying type diagnosed as neurasthenia at the time of the menopause, and operation has been followed by complete restoration to health and well-being.

\section{Observations on Dr. Moorhead's Case}

To revert to Dr. Moorhead's case, I wish to emphasize several facts that are commonly present in the type of case with which I am dealing.

1. The patient had borne children shortly after marriage, and then passed a long period of sterility, continuing until the time of the menopause. One of her confinements was complicated by a septic infection which ultimately cleared up, and although the uterus remained in retroversion her pelvic state did not apparently influence her health adversely. This condition is a basic factor in the development of the subsequent disturbances, but, owing to the remoteness of its acute period and the long interval of apparently good health, it is quite forgotten and overlooked at the time of the menopause. It naturally occurs most commonly in women who have borne children, but is not restricted to them. It may develop in childless women, or in women who have never had any complication in their confinements, as a terminal chronic state of other septic infections of the cervix, parametrium, and uterine appendages, and-as in the two cases recorded above-as the result of the presence of fibroids of the uterus, which in themselves were causing little disturbance.

2. The patient, from the time she reached the age of 43 or 44 , and so had entered that stage of existence usually termed " the period of the change of life," had serious responsibilities cast upon her, which induced her to take little notice of her own state of health and to disguise her disabilities. From the acceptance of the common belief that these were only part and parcel of what every woman has to expect for a few years at her age, she overcame her physical deficiencies by will-power, hoping that in a few years she would once more be fit. This again is a common incident in the life of many women as they reach the forties. Family, social, or assumed duties have increased, and they are reluctant to admit that these are burdensome. They feel that to do so would be to admit old age. They see many other women older than themselves easily able to do what they find burdensome, and therefore they disguise the facts, but frequently develop an irritable and morbid temperament, although fulfilling-and to the outside observer well able to fulfil--their duties.

3. The onset of the patient's illness was apparently sudden. It is a very common termination at the ages from 48 to 52 in women who have been suffering from menopausal disturbance for several years. They develop some trivial illness ; this is followed by debility altogether out of proportion to the illness, which persists in spite of treatment. The nature and intensity of symptoms vary, and lead to variation in diagnosis, which in the milder cases often ends in a final diagnosis of neurasthenia or menopause neurosis, with the deferred hope that health will be restored when the menopause is complete.

4. Six months after the patient's breakdown the monthly periods ceased, and remained absent for six months; there was little improvement as the result of this ; the general debility was slightly diminished. At the beginning of the period of amenorrhoea the possibility of a uterine origin of the whole trouble was considered, but abandoned owing to the termination of the only obvious uterine symptom and the temporary slight improvement. Uterine bleeding again started, and the patient's condition had become compatible with a diagnosis of senile dementia, incurable and progressive. Here again is an extreme degree of a common characteristic in milder cases. The women have been assured that they will be all right again when they are through the change of life, but many of them find that when the monthly periods have finally ceased their general health does not improve-rather it 
gets worse. They are then left without the hope of ultimate recovery of health, and sink into a chronic state of debility and depression, without interests, lacking in energy, a nuisance to themselves and their families, and generally spoken of as " poor So-and-so."

The case reviewed above was one of extreme physical and mental disturbance developing in association with the menopause, and the fact is established that the disturbances were terminated by the performance of a total hysterectomy. The question naturally arises, Were these symptoms due to the normal physiological changes of the menopause, or were they the effect of a pathological state in the genital organs, brought into activity by the normal physiological changes of the menopause? This question can best be answered by a review of the menopause in general, and the whole course of the active genital period of a woman's life leading up to it.

\section{The Menopause: A Conspectus}

Physical and mental alterations in the character of women are extremely common at the time of the menopause, so much so that they are looked upon as a necessary result of that change. They vary from profound and prolonged states of ill-health to slight and transient discomforts. Women suffering from these disorders seek advice, and bring themselves to the notice of their friends and the medical profession, thus setting a standard for the menopause, to the exclusion of the characteristics of that change in a large group of women who do not suffer.

If a large number of women who had reached the age of 50 years could be examined without selection, they could be divided into the following groups.

1. (a) A large group, comprising both parous and nonparous women whose monthly periods have already ceased, and who have at no time experienced any inconvenience or disturbance. They would state either that their periods stopped suddenly, having been previously normal, or that their periods had gradually diminished and ceased, to reappear slightly once or twice in the next six months and then disappear completely. Such women are invariably active, healthy, and young-looking for their age.

(b) A moderate group of women whose periods still continue with little alteration or disturbance.

2. (a) A small group of women, chiefly parous, whose periods have ceased, but who, before the complete termination, had for a year or more experienced excessive loss and resultant debility, while some had towards the end a few prolonged intervals and floodings. Such women will have returned to good health, dependent on the length of time that has elapsed in which they can recuperate.

(b) A moderate group of women whose periods persist but are, and have been for some years, heavier than normal. They suffer varying degrees of ill-health and loss of energy. Many of these women will follow the course of those above, but quite a large number will follow the course of those in Group $3(b)$.

3. (a) This is a very small group-chiefly persons whose periods have ceased after several years' disturbance and general ill-health, but whose ill-health has persisted or even increased.

(b) A group of women whose periods, after several years of excess, have become irregular and often prolonged, and whose general health is seriously degenerated. The aberration of the menstrual cycle will call for attention; the menopause will be completed by operation, or degeneration will progress and terminate under various nomenclatures, or even fatally.
4. In addition to these groups there would be a number of women who, on account of marked disturbance in the menstrual function after the age of 40 , were found to have tumours or other gross pathological conditions of the genital organs, and in whom the menopause was induced by operation.

It seems unreasonable to assume that all the first three groups are merely variations of a normal physiological process, while the need for operative treatment arose in the fourth group from an accidental pathological lesion unassociated with the menopause. It appears more logical to conclude that the characters exhibited in Group 1 are those of the normal physiological process. Those of Group 2 are slight aberrations from the normal, induced by pathological lesions; while those in Group 3 are definitely the outcome of pathological lesions quite as much in need of treatment by operation as was found necessary in Group 4. A review of the child-bearing period of a woman's life seems to justify this assumption.

The age of puberty may to some extent be compared to the age of the menopause-they come at the extremes of a woman's reproductive life. In the one there is a hypertrophic change in healthy tissues, in the other an atrophic change, often in previously damaged tissues. Taking large groups of girls-for example, in schools-it is quite an exceptional event to find marked physiological or psychological disturbances developing at the age of puberty, and such cases as do occur are nearly always found to be due to some definite pathological condition or gross developmental abnormality. The vast majority exhibit progressive development for two or three years without any disturbance in general health. By analogy it seems reasonable to suppose that the atrophic tissue changes taking place at the menopause should be spread over a period of years; that slight variations in the local manifestations of cessation should occur; that the atrophy should continue over a period of one or two years ; and that menstruation should finally cease without any or with only slight disturbance. A very large number of women do follow this course, but, as the menopause is a process of atrophy, and in many cases the organs have suffered damage or change from the normal state, a large number will exhibit faults in the physiological process.

\section{The Age of the Menopause}

The age of women when the menopause is completed, as defined by the cessation of monthly periods, ranges from 40 to 55 years, but a number of facts seem to indicate that the atrophic changes commence in the vast majority of women within fairly restricted limits of age, probably from 42 to 46 years. How long the atrophy will take to become complete varies, and depends upon the physiological state of the health of the organs.

On investigation of the ages of 1,700 women confined in the Rotunda Hospital, it was found that only thirteen women were 43 , eight 44 , and five 45 years of age. There was no woman over 45. During seven years, out if 12,500 confinements, there was only one woman aged 47 . These women are of a class who do not avoid pregnancy; and, even allowing for the attrition of advancing years, the falling-off in the power to conceive after the age of 45 is too great, and indicates some change preclusive of conception. That this change is one involving the uterus and endometrium rather than the ovaries and the process of ovulation is suggested by the facts that the sexual habits and desires of women are not diminished at the time their power to conceive has ceased, and that at operation on women at this age the ovaries are usually found normal in appearance and containing normal corpora lutea. In an analysis ${ }^{1}$ of cases of fibroids of 
the uterus treated by operation in the Rotunda Hospital during seven years, it was found that the majority developed symptoms calling for attention about the age of 45 , although other facts indicated that the fibroids had been in existence for upwards of ten years. This suggests that the fibroids are not the cause of the symptoms, but that the symptoms are the result of some change which develops about the age of 45 in the surroundings or the nutrition of the tumours.

The above facts seem to indicate that as puberty normally occurs between the ages of 13 and 16 , so the menopause normally begins between the ages of 42 and 46, and should normally be completed in two to three years, without disturbance of the general health.

\section{The Size of the Uterus}

The fully developed nulliparous uterus is described as an organ three inches long with a cavity of two and a half inches, and after childbirth it is supposed to retain a general increase in size. This latter statement is correct in a very large number of instances, but that it is a normal outcome of childbirth is doubtful. If a woman who has had an easy spontaneous labour without puerperal complications is examined six months later, and the uterus is found in a normal anteverted position, the latter will present no appreciable difference from the nulliparous organ-the external os is slightly relaxed, but the cervix is no more bulky, and the cavity is only two and a half inches long. Such women are not often examined except in immediate association with a pregnancy. During the menopause, and for some years after the termination of menses, the uterus undergoes atrophy and a considerable reduction in size. How long this change takes to be completed in a normal case has never been even approximately estimated, nor has it been established to what size a normal uterus should return. That the body of the uterus undergoes a marked diminution in size is clinically evident, and also that the vaginal portion of the cervix ceases to exist, ${ }^{2}$ while the narrowing of the vaginal vault indicates that the supravaginal portion of the cervix is also reduced. The cavity of the uterus is smaller in all directions. This would appear to be the normal course.

\section{Criteria of Normal Menopause}

The normal character of the menopause and the condition of the uterus may be thus summarized.

1. The menopause consists of an atrophy of the genital organs from the developed staté adaptable to childbearing to a state closely resembling that of the organs preceding puberty.

2. This change should occur without either physiological or mental disturbance.

3. It normally commences between the ages of 42 and 46, and is completed within two or three years. There are normal exceptions on both sides of these ages.

4. The uterus at the beginning of the menopause should be little, if at all, larger than the uterus of a young nulliparous woman-that is, three inches in length, with a proportion of body to cervix of two to one, and the cavity two and a half inches long.

5. When the atrophy of the menopause is complete, the uterus is considerably reduced in size in all its parts, being two to two and a half inches long, with a cavity one and three-quarters to two inches in length and no vaginal portion of cervix.

\section{Pathology of Uterus at the Menopause}

Women at the time of the menopause may be divided into three groups-virgin, nullipara, and para. are all liable to have developed pathological conditions during the child-bearing period, but with increasing frequency in the order of these groups. All groups may have congenital lesions, but these do not influence the menopause-with the exception of retroversions. Retroversion in some nulliparous women appears to produce in the process of time a hyperplasia of the uterus. This is probably the result of excessive congestion at the menstrual periods causing excessive flow, which, associated with defective drainage, stimulates uterine action and produces muscle hypertrophy.

In the group of virgins the incidence of fibroids is the chief factor ; these are always associated with hypertrophy of the uterine muscle, and may in time be extruded outside the muscle, becoming subserous. The muscle may undergo involution, but if this does not occur the uterus remains in a state of hyperplasia at the time of the menopause. If the fibroids become submucous they produce discharge and promote the tendency to chronic infection which frequently involves the cervix, causing chronic cervicitis. The secondary results of retroversion also lead to chronic infection of the same sort, as shown by the common association of erosion. There are thus in the virgin two common potent causes of metritis and cervicitis which are of long standing and persist at the time of the menopause.

In the nulliparous group there is added the common incidence of ascending infections, independent of either fibroids or displacement.

In the parous group the risk of acquired lesions is enormously increased. Besides all those of the other groups, there is the frequent occurrence of acquired or post-partum retroversion. In an investigation of 100 women after normal uncomplicated confinements, twenty-seven were found to have retroverted uteri six weeks post partum, and nine of these had retroversion before they became pregnant. Retroversion of the uterus, whether acquired or a return to the congenital condition, is practically always associated with subinvolution, which persists and becomes a chronic hyperplasia if the retroversion is not corrected while the subinvolution is in an acute or subacute stage. This practice is far from common, and the retroversion is allowed to persist. It is a common cause of one-child sterility; if it occurs once it will recur after all subsequent pregnancies. Although it does not often cause symptoms during the lying-in period, it develops about the ninth day, and seriously interferes with the free drainage of the uterus during the remainder of the puerperium. It is a potent cause of low chronic infection, productive of chronic metritis and cervicitis. Lacerations of the cervix, especially those involving tissue above the vaginal portion, and the devitalizing effect of prolonged pressure on the cervical tissues in protracted labour, promote the chances of infection which, although of a low type calling for no observation during the puerperium, is the cause of the indurated enlarged cervices so commonly found in parous women.

The causes of pathological states of the uterus at the time of the menopause may be summed up as follows: (1) ascending infection promoted by stagnation of menstrual or other discharges from the uterus and cervix; (2) laceration and damage during childbirth of the cervix and parametric tissues, exposing those tissues to chronic infection; (3) septic infection following childbirth; (4) gonococcal infection upon which secondary infection has supervened-far less common than the preceding; and (5) growth and influence of neoplasms, chiefly uterine fibroids. The pathological lesions that are produced and influence the course of the menopause are: (1) chronic hyperplasia of the body of the uterus; (2) chronic 
metritis with chronic cervicitis ; and (3) chronic cervicitis, with or without parametric induration. Associated with these conditions there is an increased vascularity; the smaller arteries are hypertrophied, and there is venous congestion.

During the active child-bearing period of life these lesions produce varying degrees of leucorrhoea calling for palliative treatment, such as routine douching, applications of caustics, and curettage. These treatments have no more curative effect upon the chronic infection than gargles in chronic tonsillitis. Amputations of the cervix effect cures when the portio vaginalis is the part involved, but they frequently stop far short of removing the main centre of infection, which is the supravaginal portion. When the period of the menopause is reached, and atrophy of tissue has proceeded, the vitality of the tissues is reduced, and the production of toxins from the chronic infection is increased. The local condition, leucorrhoea, has been accepted as inevitable, while increased menstrual loss reduces the woman's resistance, but is considered a necessary part of the menopause.

\section{Effect of Pathological Lesions on the Menopause}

The simplest lesion is chronic hyperplasia, commonly the result of post-partum retroversion or associated with fibroids. The over-sized organ of this lesion has to undergo atrophy. There is therefore a prolongation of the process, with excessive loss at the periods and resulting debility, but as a rule the completion of the process is not prevented. When the atrophy is finished the uterus remains larger than it should be, being about the size of a nulliparous organ, and the woman recovers from the temporary debility. Sometimes the menstrual losses are so profuse as to call for treatment, and curettage is found to give temporary relief, which helps to tide matters over. In others the loss is sufficient to call for more active treatment, and excellent results are obtained by $x$ rays or radium. This is the lesion that exists in the second group of women previously described as seen at 50 years of age.

The most pronounced lesion is chronic metritis with chronic cervicitis. There is always hyperplasia; this varies in amount, and is often very marked. This lesion during the process of atrophy produces all the symptoms due to hyperplasia and varying in proportion with it, but the symptoms are increased by the added element of toxic absorption. When the atrophy is complete the menses may cease or become irregular, but the general health does not improve, and often further deteriorates. This is the lesion that exists in the third group of women at 50 years of age. Various treatments, short of total hysterectomy, are resorted to for the menopause, and are credited with being of benefit if the menses terminate irrespective of the permanent loss of fertility in the women quite inconsistent with their age.. These are the cases that do not give satisfactory results with $x$ rays and radium. This is to be expected, as they contain the chief contraindication to this treatment-septic infection. As this is not obvious it is overlooked, and this lesion is only excluded from $x$-ray treatment when it is accompanied by evidence of chronic pelvic peritonitis, parametritis, and adhesions.

The third lesion-chronic cervicitis-may be associated with simple hyperplasia, when the local symptoms referable to that will be present and follow the course already described, but the general symptoms will be increased. When hyperplasia is not present the menopause, so far as the menstrual cycle element is concerned, is passed uneventfully; but there is an unaccountable element of not well-being. This not well-being continues after the menses have ceased, and may ultimately die out with no permanent ill effects ; but many women show premature ageing, and if the infection does not die out their illhealth persists. Women with this lesion come under Groups $2(a)$ and $3(a)$ at the age of 50. Of the various treatments adopted for this condition, $x$ rays and radium have the same influence as in the other lesions, but diathermy needs to be especially mentioned. Its application may hasten and even promote the dying out of the infection when this is limited to the portio vaginalis; but when the infection extensively involves the cervix, and especially when the parametrium is affected, its action is not sufficiently penetrating.

\section{SUMMARY}

All through this paper reference to any individual symptom of the menopause has been avoided; as a whole they are too various and undefined, with the exception of flushings and sweating. These two are much the least important; they are probably as near as an abnormality can be to a natural incident. They are probably wholly of ovarian origin, and occur in women who pass through an absolutely normal menopause ; but in these women they more often arise after the menses have ceased, and are seldom sufficient to call for more than transient notice, and an assurance that nothing serious is wrong. It is when they are associated with the conditions considered in this paper that they are increased and call for serious comment, and are so often intractable to treatment. They respond to all sorts of treatments which improve the general health, and relapse when the general health deteriorates.

The writer is in no sense advocating hysterectomy as a universal treatment for ill-health at the time of the menopause. The differential diagnosis of the lesions described is a difficult and minute matter. It is seldom possible to make an early decision-such cases are mentioned in Group 4 of women at 50. In the majority of instances a decision will be reached only after a period of observation, but the lesions which exist at the onset of the menopause can be observed, and should be sufficient to indicate treatment by hysterectomy if the illhealth increases. Serious and permanent damage may thus be prevented. When hysterectomy is resorted to it must be of the total type. I believe that the failure to observe the benefits of hysterectomy in these cases has in the past resulted from the common practice of performing the high partial operation: this relieved the menstrual symptoms, but did not remove the toxic element.

\section{Conclusions}

1. The menopause should occur with as little disturbance as puberty.

2. During the child-bearing period of life many women acquire pathological lesions of the uterus which frequently induce chronic bacterial infection of the uterine body and cervix.

3. When the atrophy of the menopause occurs the toxic influence of the infection is stimulated.

4. The pathological lesions delay and disturb the normal course of the menopause.

5. The toxic absorption causes ill-health, and frequently persists after the menopause is completed.

6. If the aberration of the menopause or the toxic effects are severe, treatment by total hysterectomy is indicated to prevent permanent damage to the future health.

\section{References}

1 FitzGibbon: Irish Journ. Med. Sci., December, 1928.

- Fairbairn: Gynaecology and Obstetrics, 1924, 658. 